\title{
Diagnosing pseudoaneurysm of the gastroduodenal artery on endoscopic ultrasound
}

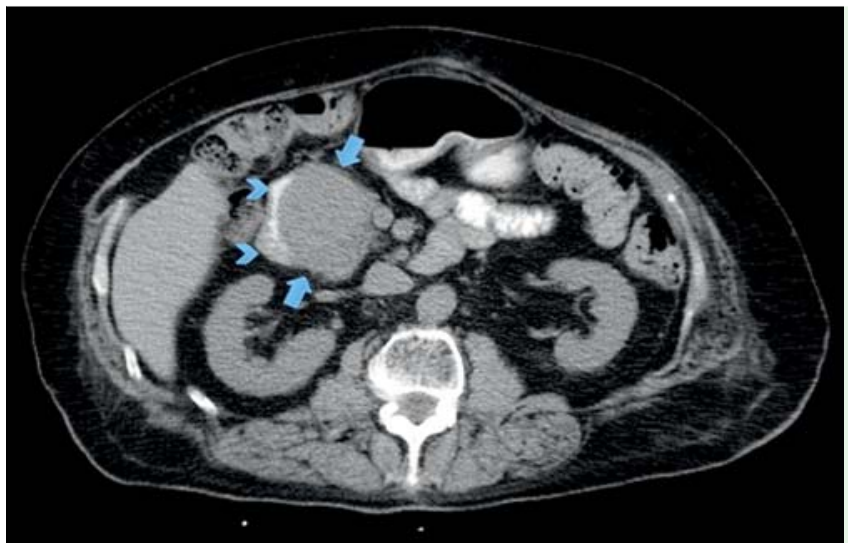

Fig. 1 Computed tomographic scan of the spine without contrast in a 72 -year-old man presenting with acute-on-chronic back pain incidentally showing a mass (arrows) compressing the second part of the duodenum, which has contrast in the lumen (arrowheads).
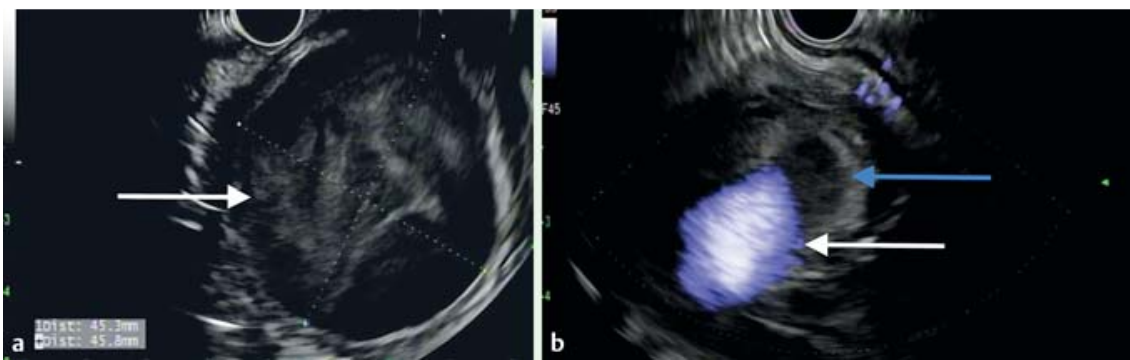

Fig.3 a Endoscopic ultrasound showing a heterogeneous mass (arrow) posterior to the duodenum. b The high blood flow rate (white arrow) in the mass (blue arrow) is indicative of aneurysm.

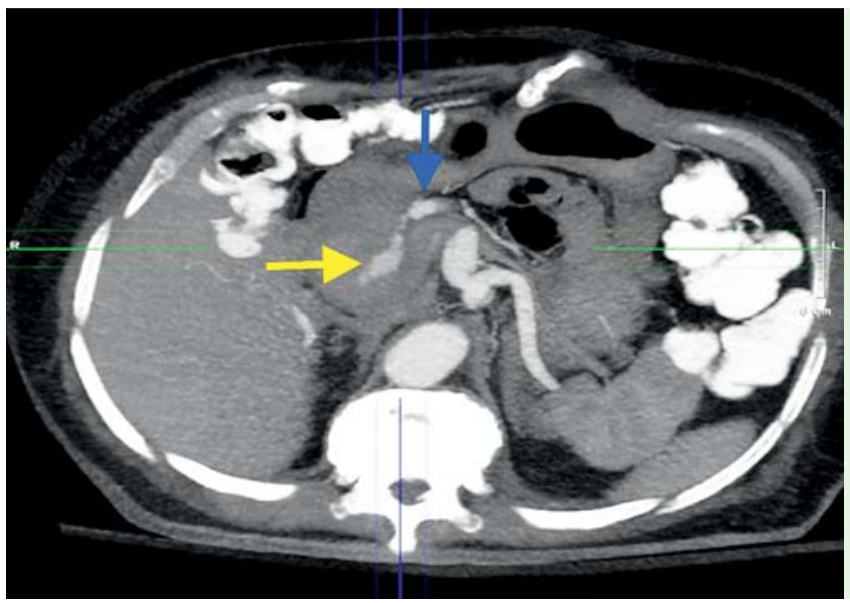

Fig. 4 Computed tomographic angiogram of the abdomen showing the gastroduodenal artery (blue arrow) with extravasation of contrast into the pseudoaneurysm (yellow arrow). Blood clot is noted in the periphery of the pseudoaneurysm, with compression of the duodenum.

A 72-year-old man presented to the emergency room with acute-on-chronic back pain. His medical history was significant for cholecystectomy, deep venous thrombosis, multiple myeloma, and angioedema with the administration of iodinated contrast. He was on long-term enoxaparin and weekly steroid therapy.

Computed tomography of the spine revealed compression fractures of the T11, T12, and L2 vertebrae due to multiple myeloma. A $5.3 \times 4.7-\mathrm{cm}$ mass was noted incidentally in the second portion of duodenum and initially diagnosed as an intramural hematoma ( $\bullet$ Fig. 1 ). The mass was seen to have increased in size (to $5.6 \times 4.9 \mathrm{~cm}$ ) on a noncontrast computed tomographic scan obtained for followup of the mass 2 months later.

Upper gastrointestinal endoscopy showed extrinsic compression of the bulb and second portion of the duodenum without

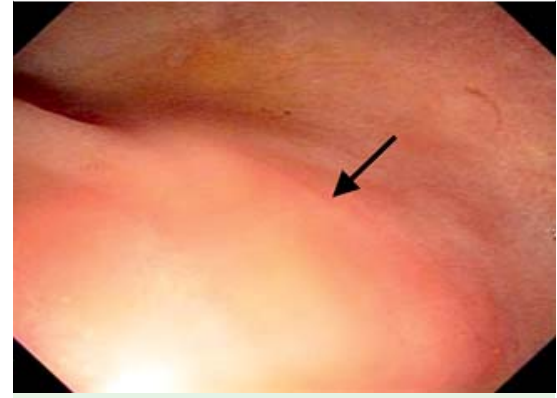

Fig. 2 Upper gastrointestinal endoscopy showing extrinsic compression of the duodenum (arrow) without any mucosal lesions.

any mucosal lesions ( $\bullet$ Fig. 2). Endoscopic ultrasound was done right away and revealed a $4.5 \times 4.5-\mathrm{cm}$ round vascular lesion with a high blood flow rate posterior to the duodenal bulb, highly suggestive of a gastroduodenal artery aneurysm ( $\bullet$ Fig.3). Computed tomographic angiography of the abdomen after premedication with intravenous glucocorticoids confirmed a $5.9 \times 6.4 \times 6.9-\mathrm{cm}$ pseudoaneurysm of the proximal branch of the gastroduodenal artery compressing the second portion of the duodenum ( $\bullet$ Fig. 4 ).

Metallic coils were successfully placed proximally and distally to the pseudoaneurysm by interventional radiology, with no detectable blood flow after the coiling ( Fig.5). Follow-up computed tomographic angiography of the abdomen after 7 weeks showed a decrease in the size of the pseudoaneurysm and no extravasation of contrast.

A few cases of visceral artery pseudoaneurysm treated with the injection of drugs under endoscopic ultrasound (EUS) guidance have been reported [1 - 3]. However, this is the first case in which EUS was instrumental in establishing a diagnosis of gastroduodenal artery pseudoaneurysm because of a patient's known allergy to iodinated contrast. Cholecystectomy was the only identifiable risk factor for the patient's gastroduodenal artery pseudoaneurysm.

Endoscopy_UCTN_Code_CCL_1AF_2AD

\section{Competing interests: None}




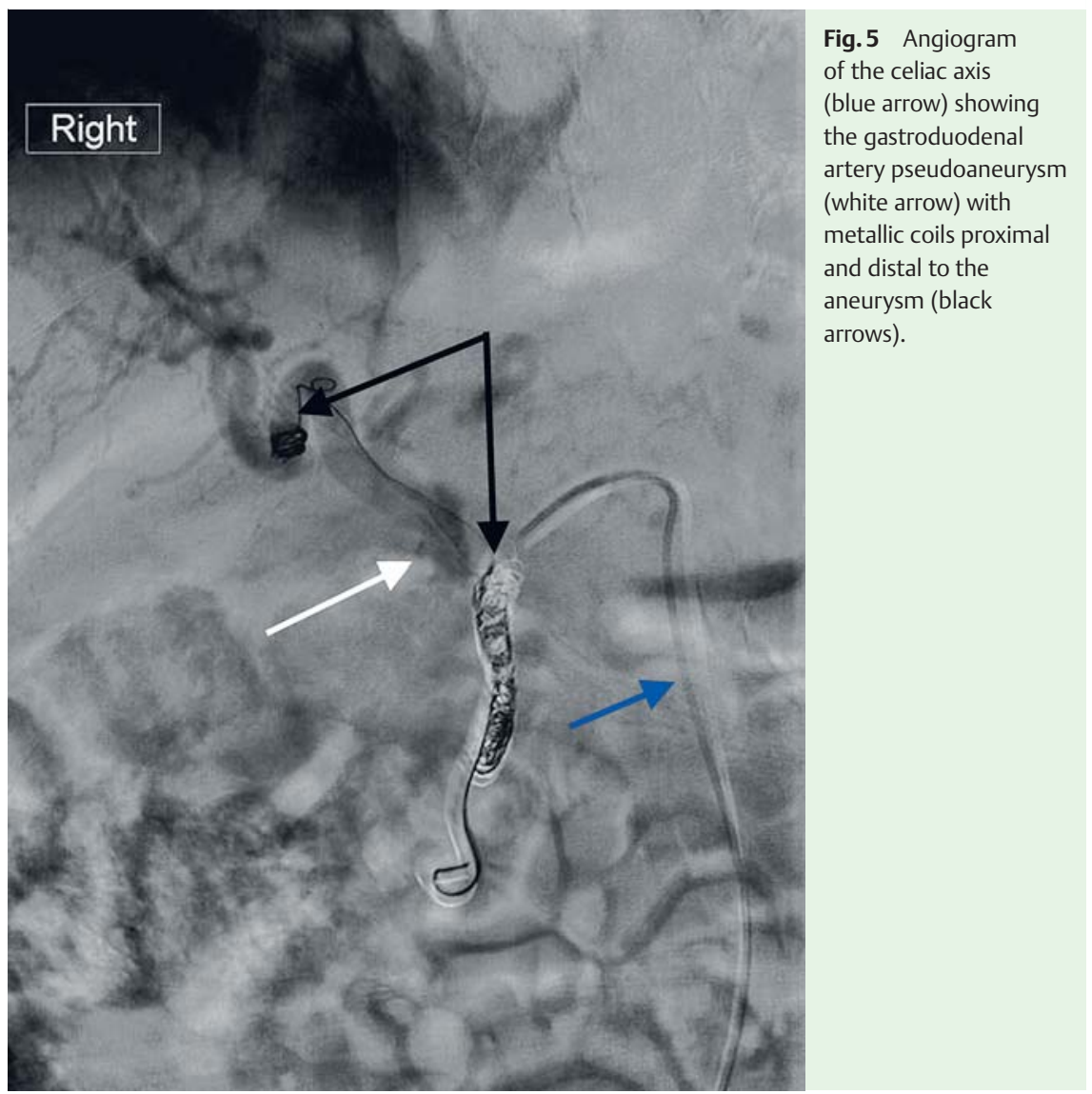

\section{Avin Aggarwal', Shashank Garg ${ }^{2}$}

${ }^{1}$ Department of Medicine, Hennepin County Medical Center, Minneapolis, Minnesota, USA

${ }^{2}$ Division of Digestive Health and Nutrition, Department of Internal Medicine, University of Kentucky College of Medicine, Lexington, Kentucky, USA

\section{References}

1 Roberts KJ, Jones RG, Forde $C$ et al. Endoscopic ultrasound-guided treatment of visceral artery pseudoaneurysm. HPB (Oxford) 2012; 14: 489-490

2 Habib N, Hassan S, Abdou R et al. Gastroduodenal artery aneurysm, diagnosis, clinical presentation and management: a concise review. Ann Surg Innov Res 2013; 7: 4

3 Bokun T, Grgurevic I, Kujundzic M et al. EUSguided vascular procedures: a literature review. Gastroenterol Res Pract 2013; 2013: 865945

\section{Bibliography}

DOI http://dx.doi.org/

10.1055/s-0034-1392614

Endoscopy 2015; 47: E404-E405

(c) Georg Thieme Verlag KG

Stuttgart · New York

ISSN 0013-726X

\section{Corresponding author \\ Shashank Garg, MBBS}

Division of Digestive Health and Nutrition Department of Internal Medicine

University of Kentucky College of Medicine 800 Rose Street

Lexington, Kentucky 40536

USA

Fax: +1-859-257-9287

shashank.garg@uky.edu 